\title{
COMPOSICIÓN FlORÍSTICA Y DIVERSIDAD DE LA VEGETACIÓN DE HUMEDALES EN LOS LAGOS INTERDUNARIOS DE VERACRUZ
}

\author{
Luis Alberto Peralta-Peláez ${ }^{1,3}$ y Patricia Moreno-Casasola ${ }^{2}$ \\ ${ }^{1}$ Instituto Tecnológico de Veracruz, Departamento de Ingeniería Química y Bioquímica, \\ Av. Miguel Ángel de Quevedo 2779, Col. Formando Hogar, 91860, Veracruz, Veracruz, México. \\ ${ }^{2}$ Instituto de Ecología, A.C., Depto. de Ecología Funcional. km 2.5 carretera antigua a Coatepec no. 351, \\ Xalapa 91070, Veracruz, México. \\ ${ }^{3}$ Autor para la correspondencia. Correo-e: peralta@itver.edu.mx
}

\begin{abstract}
Resumen: Los lagos interdunarios de la costa de Veracruz, México, son cuerpos de agua dulce, someros, que presentan condiciones favorables para el establecimiento de humedales herbáceos y arbóreos. Las dunas que los rodean han sido transformadas en potreros introduciéndose especies forrajeras y caña de azúcar. Nuestro objetivo es describir y comparar la estructura, composición y diversidad de la vegetación de humedales de 15 lagos interdunarios en la zona rural. Se tomaron en cuenta factores ambientales como pH, salinidad, tamaño, profundidad y número de meses secos. Se registraron 46 familias (27 de las cuales son acuáticas estrictas) y 82 especies, entre ellas Sagittaria lancifolia, Pontederia sagittata, Ceratophyllum demersum. La riqueza fluctuó entre 5 y 33 especies. La clasificación encontró nueve grupos florísticos en los humedales de estos lagos, y en tres de ellos aparecen especies de pastos usados en la ganadería (Cynodon dactylon, Echinochloa pyramidalis). El principal eje de variación en la ordenación separó a los lagos en función del pH y del número de meses que permanecen secos. Su conservación y uso sustentable requieren de planes de manejo conjuntos con los usuarios.

Palabras clave: Diversidad, humedales, lagos interdunarios, lagos someros, vegetación acuática.
\end{abstract}

\begin{abstract}
Dune lakes of the coast of Veracruz, Mexico, are shallow freshwater lakes with suitable conditions for the establishment of herbaceous and arboreal wetlands. Most of the vegetation of the surrounding dunes has been transformed into pastures for grazing lands and sugar cane plantations. The objective of this paper is to describe and compare structure, composition and diversity of the wetland vegetation of 15 dune lakes in the rural area. Environmental parameters ( $\mathrm{pH}$, salinity, size, depth and number of dry months) were taken into account. Plant diversity of these lakes comprise 46 families (27 strictly aquatic) and 82 species, among them Sagittaria lancifolia, Pontederia sagittata, Ceratophyllum demersum. Species richness fluctuated between 5 and 33 species. The classification resulted in nine floristic wetland groups, three of them with grass species from the surrounding areas $(C y n o d o n$ dactylon, Echinochloa pyramidalis). Ordination separated lakes based on $\mathrm{pH}$ and number of dry months. Management plans should be developed together with the owners to maintain both the use of these lakes and the composition and structure of their wetlands.
\end{abstract}

Key words: Diversity, wetland, dune lakes, shallow lakes, aquatic vegetation.

$\mathbf{E}_{\mathrm{d} e}$ Golfo de México se caracteriza por un fuerte transporte de sedimentos por corrientes de agua y vientos capaces de mover arena y formar grandes médanos (Moreno-Casasola y Espejel, 1986). En la región correspondiente al norte de México, la línea de la costa está orientada de norte a sur, igual que los vientos dominantes que transportan arena, permitiendo la formación de grandes sistemas de médanos (Moreno-Casasola, 1982, 2004) con depresiones inundadas temporal o permanentemente, formando hondonadas (sensu Ranwell, 1972) y lagos interdunarios, respectivamente.

Estos hábitats se localizan desde Tamaulipas hasta Ta- basco, pero principalmente en la zona media de Veracruz, entre los municipios de Alto Lucero y Boca del Río, que coincide con las grandes extensiones de médanos del centro de Veracruz. Los lagos que se localizan en los municipios de Boca del Río y Veracruz están inmersos en el crecimiento urbano (Siemens et al., 2006) y forman parte del sitio Ramsar Lagunas Interdunarias de Veracruz. Los lagos interdunarios rurales, objeto del presente estudio, no están bajo protección.

Este tipo de lagos se encuentran ampliamente distribuidos en otras zonas costeras del mundo (Leentvaar, 1997; Wetzel, 1981). Sin embargo, la mayoría de los estudios se 
han realizado en Estados Unidos (Tiner, 2003), Gran Bretaña (Ranwell, 1972), Sudáfrica (Boorman et al., 1997; Leentvaar, 1997) y sobre todo Holanda y Alemania (Lammerts y Grootjans, 1998; Sival y Lammerts, 1997; Sival y Strijkstra-Kalk, 1997; van der Meulen y Naber, 1997; Leentvaar, 1997 y De Bruyn, 1997, entre otros), en los trópicos, en Brasil están los trabajos de Domínguez y Bittencourt (1994), Queiroz-Matias y Paula-Nunes (2001). En el caso de México, Martínez et al. (1997), Moreno-Casasola y Vázquez (1999) y Vázquez (2004) han abordado principalmente el estudio de la vegetación de depresiones con inundación temporal, mientras que Yetter (2004) estudió la hidrología de un conjunto de humedales en los que se incluye este tipo de lagos, determinando que las principales entradas de agua son a través del manto freático de fuentes tanto cercanas como distantes así como por lluvias. Sarabia (2004) estudió la química del agua de los lagos interdunarios de la ciudad de Veracruz, mientras que Peralta-Pélaez et al. (2007) realizaron estudios de diversidad de insectos acuáticos en varios lagos interdunarios de la región de trabajo.

En las depresiones húmedas e inundadas de estos sistemas de dunas del Golfo de México se dan condiciones favorables para la germinación y establecimiento de plantas y son zonas donde se inicia la colonización y estabilización de estos sistemas (Moreno-Casasola y Vázquez, 1999). Aunque los suelos de estas áreas son arenosos y bajos en nutrientes, la humedad y/o inundación favorecen el crecimiento de plantas y permiten que lentamente se incorpore materia orgánica mejorando su fertilidad y textura. Los lagos interdunarios y sus humedales prestan servicios ambientales en la región, como zonas de recarga de mantos freáticos, depuración de aguas provenientes de la zonas agrícolas, áreas para la pesca, zonas de alimento, estancia y anidación para un gran número de aves migratorias, por mencionar algunos (Leentvaar, 1997; Tiner, 2003; Grootjans et al., 2004). En México estos sistemas son utilizados como abrevaderos de ganado y para cultivo de tilapia; además los sistemas de dunas y las depresiones mas húmedas que los rodean, se utilizan de manera importante para actividades ganaderas y siembra de caña de azúcar (observación personal).

En estos lagos se forman humedales de especies herbáceas enraizadas y flotantes, así como arbóreas, que forman comunidades diversas que se establecen tanto en las orillas como en las zonas centrales de estos ambientes poco profundos. En México únicamente existe información sobre los humedales costeros de agua dulce establecidos en planicies

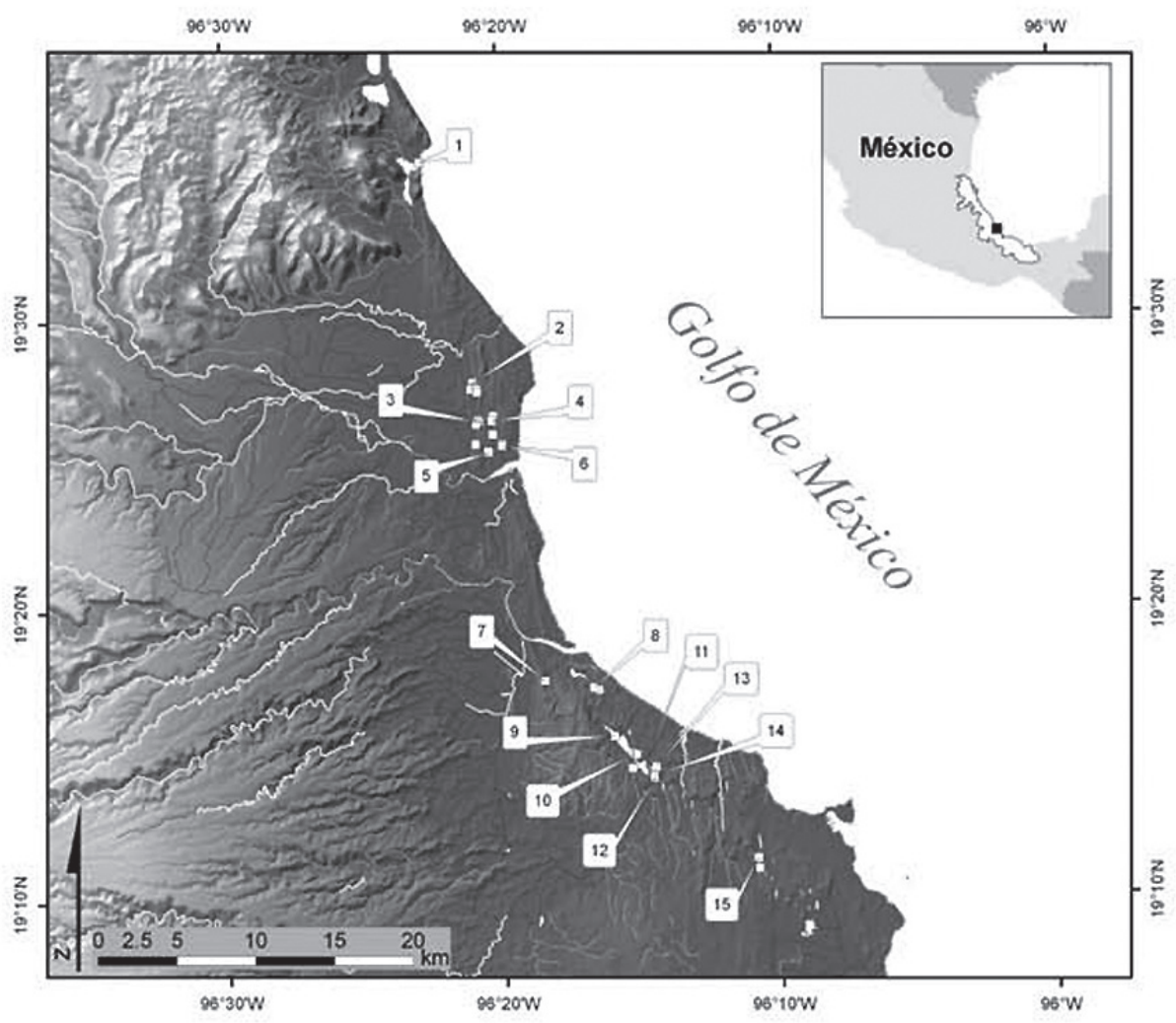

Figura 1. Localización de los lagos interdunarias en la región central del estado de Veracruz, México, 1) CICOLMA (LM), 2) Don Gil (DG), 3) Felix (Fel), 4) Jagüeyes (Jag), 5) Tolega (Tol), 6) Ojite (Oj), 7) Catalana (Cat), 8) Colorada (Col), 9) San Julián (SJ), 10) La Palma (LP), 11) Minerva (Min), 12) Zendejas (Zen), 13) Abascal (Abs), 14) Manguito (Man), 15) Las Conchas (Con). Los tonos de grises indican las subcuencas: Actopan, La Antigua Jamapa 
de inundación (Lot, 1991; Lot et al., 1998; Lot et al., 1999), pero no se ha investigado la vegetación presente en estos ambientes.

El presente estudio tiene como objetivo describir y comparar la composición, estructura y diversidad de la vegetación de los humedales de quince lagos interdunarios que se localizan en una zona rural de la planicie costera de la región central de Veracruz, sobre el Golfo de México.

\section{Método}

Área de estudio. La zona de estudio se localiza en la franja de dunas costeras de la llanura costera del Golfo de México en el centro del estado de Veracruz $\left(19^{\circ} 35^{\prime} 12^{\prime \prime} \mathrm{N}\right.$ y $96^{\circ} 22^{\prime}$ $18^{\prime \prime} \mathrm{W} ; 19^{\circ} 10^{\prime} 53.0^{\prime \prime} \mathrm{N}$ y $96^{\circ} 10^{\prime} 53.3^{\prime \prime} \mathrm{W}$ ) (figura 1). La zona presenta clima tropical subhúmedo con lluvias en verano (Aw), una precipitación media anual de $1550 \mathrm{~mm}$ y una temperatura promedio anual de $26^{\circ} \mathrm{C}$ (García, 1981). Para la época de lluvias (junio a octubre), la precipitación promedio mensual y temperatura es de $224( \pm 25) \mathrm{mm}$ y $30^{\circ} \mathrm{C}( \pm 3.5)$ y para la época de secas es de $44( \pm 37) \mathrm{mm}$ y $22^{\circ} \mathrm{C}( \pm 3)$. El nombre de los lagos corresponde a la nomenclatura publicada en los mapas y cuando no se encontró se usó el nombre local o el del propietario donde se localizan.

Para ubicar y delimitar la zona de estudio y las actividades productivas, se utilizaron fotos aéreas de 1999 a una escala de 1:70000. Se digitizaron y se usaron para calcular la superficie de cada lago.

Muestreo. Se muestreó y colectó en 15 lagos interdunarios (figura 1) en la vegetación presente al final de la época de lluvias (octubre-noviembre 2003) y secas (marzo-mayo 2004). Se muestreó en cuadros de $2 \times 2 \mathrm{~m}^{2}\left(10 \times 10 \mathrm{~m}^{2}\right.$ para los árboles) a lo largo de dos transectos en cada lago. El muestreo fue estratificado, tomándose un conjunto de muestras en la zona comprendiendo las márgenes y otro conjunto en el cuerpo de agua o parte central de cada lago. Los cuadros se establecieron cada cinco metros sobre cada uno de los transectos. En cada cuadro se anotó la lista de especies y se calculó la cobertura total de la vegetación y la cobertura de cada una de las especies presentes utilizando la escala de cobertura-abundancia de Westhoff y van der Maarel (1978). No se tomaron datos de densidad debido a la predominancia de formas de crecimiento que no permitían distinguir entre individuos. El número total de muestras varió en función del tamaño del lago y de las comunidades presentes, con un mínimo de 12 cuadros a un máximo de 68 . Los ejemplares botánicos de respaldo se depositaron en los herbarios XAL (Instituto de Ecología A.C.) y MEXU (Instituto de Biología, UNAM).

Análisis de datos. Los datos de cobertura se transformaron a datos de valor de importancia relativa (VIR) por especie: $V I R=\left[(\mathrm{FR}+\mathrm{CR})(2)^{-1}\right] * 100$ donde la frecuencia relativa (FR) se definió como el número de cuadros donde aparece la $\mathrm{Sp}_{\mathrm{i}}$, entre el número de cuadros muestreados. La cober- tura relativa $(\mathrm{CR})$ se consideró como la cobertura promedio de la especie i en los cuadros donde aparece, dividido por la cobertura máxima que puede alcanzar la misma especie.

Se tomaron datos de conductividad, salinidad y $\mathrm{pH}$ a lo largo de cinco transectos, cuatro de ellos orientados a lo largo de los puntos cardinales (Norte, Este, Sur y Oeste) y el último al centro de todos ellos, intentando que coincidiesen con las diferentes comunidades vegetales muestreadas. Se midieron in situ usando un multiparamétrico YSI 6600 sobre estos transectos. Asimismo, en cada lago se midió la profundidad en el centro durante la época de lluvias y la de secas, utilizando una varilla marcada en centímetros.

Para evaluar el esfuerzo de muestreo se realizaron curvas de acumulación de especies por lago, con el estimador Jackknife de primer orden (Peet, 1975) utilizando el programa EstimateS ver. 07, (Colwell, 2005).

Análisis de datos. Los datos se analizaron mediante métodos multivariados con el fin de definir agrupaciones en función de similitudes florísticas y ver la distribución de los lagos en función de la vegetación y los gradientes ambientales. Se usaron dos matrices de vegetación. En la primera de ellas se utilizaron 330 cuadros de muestreo de vegetación y 68 especies, provenientes de los 15 lagos. En este caso se eliminaron las especies que solamente aparecieron en uno o dos cuadros de vegetación. En la segunda matriz de vegetación se hizo una síntesis obteniendo el valor de importancia de cada especie en cada lago, construyendo una matriz de 15 sitios y 82 especies, ya que no se eliminó ninguna especie. En ambos casos se transformaron los datos por medio del método de Beals Smoothing (Mc Cune y Grace, 2002). Para entender la gran variabilidad florística de las muestras se aplicó un análisis de clasificación. Se buscó establecer agrupaciones florísticas con base en el método de clasificación jerárquica denominado Beta Flexible con un valor de $\beta$ $=-0.25$, y la medida de distancia de Sorensen (Bray-Curtis) ya que conforme $\beta$ se vuelve negativo, el método incrementa los espacios y los elementos se agrupan de manera más intensa (Mc Cune y Grace, 2002). Como matriz secundaria se utilizaron los valores promedio de los parámetros físicos del agua en cada lago ( $\mathrm{pH}$, salinidad, tamaño, profundidad, y número de meses que permanece seco). Estos últimos datos fueron tomados del Cuadro 1. En ambas ordenaciones se empleo el método de Análisis de Componentes Principales y el programa utilizado fue PC-ORD, Version 4.25 (Mc Cune y Grace, 2002).

Análisis de diversidad. Para cada lago se estimó la riqueza de especies ( $\mathrm{S}=$ número de especies). La diversidad alfa se calculó utilizando el índice de diversidad de Shannon-Weaver, mediante el programa PC-ORD, Version 4.25 (Mc Cune y Grace, 2002), al igual que la equitatividad. Para comparar y establecer si existe diferencia en la diversidad obtenida para cada lago, se realizó la prueba de $t$ propuesta por Hutcheson (Zar, 1999; Magurran, 2004) la cual consiste en:

$\mathrm{t}=\left(\mathrm{H}^{\prime} 1-\mathrm{H}_{2}{ }_{2}\right)\left(\mathrm{SH}^{\prime} 1-\mathrm{SH}_{2}\right)^{-1}$ donde 
Cuadro 1. Características de los lagos interdunarios.

\begin{tabular}{|c|c|c|c|c|c|c|c|}
\hline Clave & Coordenadas & $\begin{array}{l}\text { Superficie } \\
\text { total (ha) }\end{array}$ & $\begin{array}{l}\text { No. de meses } \\
\sin \text { agua }\end{array}$ & $\begin{array}{l}\mathrm{pH} \text { (media } \\
\text { anual) }\end{array}$ & $\begin{array}{c}\text { Salinidad } \\
\text { (media anual, } \\
\text { partes por mil) }\end{array}$ & $\begin{array}{l}\text { Profundidad en } \\
\text { secas-lluvias } \\
(\mathrm{cm})\end{array}$ & $\begin{array}{l}\% \text { de superficie cubierta por } \\
\text { vegetación y especies } \\
\text { dominantes }\end{array}$ \\
\hline $\begin{array}{l}\text { LM (La Mancha, } \\
\text { CICOLMA) }\end{array}$ & $\begin{array}{l}19^{\circ} 35^{\prime} 25 \mathrm{~N} \\
96^{\circ} 22^{\prime} 49 \mathrm{~W}\end{array}$ & 1.5 & 0 & 7.79 & 0.240 & $0-150$ & 100- Pistia stratiotes \\
\hline DG (Don Gil) & $\begin{array}{l}19^{\circ} 18^{\prime} .295 \mathrm{~N} \\
96^{\circ} 17^{\prime} .150 \mathrm{~W}\end{array}$ & 12 & 0 & 6.22 & 0.031 & $0-150$ & $\begin{array}{l}\text { 50- Cynodon dactylon, } \\
\text { Thalia geniculata, P. stratiotes }\end{array}$ \\
\hline Fel (Félix) & $\begin{array}{l}19^{\circ} 26^{\prime} .428 \mathrm{~N} \\
96^{\circ} 20^{\prime} .892 \mathrm{~W}\end{array}$ & 8 & 3 & 6.25 & 0.044 & $0-120$ & 75- C. dactylon, T. geniculata \\
\hline Jag (Jagueyes) & $\begin{array}{l}19^{\circ} 25^{\prime} 34 \mathrm{~N} \\
96^{\circ} 19^{\prime} 564.7 \mathrm{~W}\end{array}$ & 6 & 3 & 6.50 & 0.084 & $0-100$ & 70- C. dactylon, T. geniculata \\
\hline Tol (Tolega) & $\begin{array}{l}19^{\circ} 25^{\prime} 23.3 \mathrm{~N} \\
96^{\circ} 20^{\prime} 24 \mathrm{~W}\end{array}$ & 1 & 3 & 7.57 & 0.168 & $0-120$ & 100- P. stratiotes, Mimosa pigra \\
\hline Oj (Ojite) & $\begin{array}{l}19^{\circ} 25^{\prime} .675 \mathrm{~N} \\
96^{\circ} 19^{\prime} .923 \mathrm{~W}\end{array}$ & 1 & 0 & 7.08 & 0.248 & $10-150$ & $\begin{array}{l}\text { 70- C. dactylon, Pontederia } \\
\text { sagittata, P. stratiotes }\end{array}$ \\
\hline Cat (Catalana) & $\begin{array}{l}19^{\circ} 17^{\prime} 30^{\prime \prime} \mathrm{N} \\
96^{\circ} 18^{\prime} 30^{\prime \prime} \mathrm{W}\end{array}$ & 50 & 0 & 8.03 & 0.206 & $30-200$ & 80- Typha domingensis \\
\hline Col (Colorada) & $\begin{array}{l}19^{\circ} 17^{\prime} 10^{\prime \prime} \mathrm{N} \\
96^{\circ} 16^{\prime} 30^{\prime \prime} \mathrm{W}\end{array}$ & 15 & 0 & 8.65 & 0.214 & $300-650$ & $\begin{array}{l}\text { 20- T. domingensis, } \\
\text { Nymphoides indica }\end{array}$ \\
\hline SJ (San Julián) & $\begin{array}{l}19^{\circ} 15^{\prime} 27.7^{\prime \prime} \mathrm{N} \\
96^{\circ} 15^{\prime} 47.8^{\prime \prime} \mathrm{W}\end{array}$ & 150 & 0 & 9.05 & 0.136 & $100-250$ & 40- T. domingensis \\
\hline LP (La Palma) & $\begin{array}{l}19^{\circ} 15^{\prime} 00,05^{\prime \prime} \mathrm{N} \\
96^{\circ} 15^{\prime} 18.3^{\prime \prime} \mathrm{W}\end{array}$ & 2 & 0 & 7.21 & 0.366 & $100-200$ & 50- T. domingensis \\
\hline Min (Minerva) & $\begin{array}{l}19^{\circ} 14^{\prime} 56^{\prime \prime} \mathrm{N} \\
96^{\circ} 15^{\prime} 14^{\prime \prime} \mathrm{W}\end{array}$ & 1 & 2 & 7.06 & 0.306 & $10-150$ & 90- P. sagittata, $T$. domingensis \\
\hline Zen (Zendejas) & $\begin{array}{l}19^{\circ} 13^{\prime} 59 \mathrm{~N} \\
96^{\circ} 14^{\prime} 28.3 \mathrm{~W}\end{array}$ & 1.5 & 0 & 7.11 & 0.234 & $150-250$ & $\begin{array}{l}\text { 75- T. domingensis P. sagittata, } \\
\text { Ceratophyllum demersum, } \\
\text { Utricularia gibba }\end{array}$ \\
\hline Abs (Abascal) & $\begin{array}{l}19^{\circ} 14^{\prime} .505 \mathrm{~N} \\
96^{\circ} 14^{\prime} .505 \mathrm{~W}\end{array}$ & 3 & 0 & 7.43 & 0.245 & $50-200$ & $\begin{array}{l}\text { 50- T. domingensis P. sagittata, } \\
\text { C. demersum, Utricularia foliosa }\end{array}$ \\
\hline Man (Manguito) & $\begin{array}{l}19^{\circ} 14^{\prime} .505 \mathrm{~N} \\
96^{\circ} 14^{\prime} .505 \mathrm{~W}\end{array}$ & 3 & 0 & 7.35 & 0.245 & $50-200$ & $\begin{array}{l}\text { 50- } T \text {. domingensis, P. sagittata, } \\
\text { C. demersum, U. foliosa }\end{array}$ \\
\hline Con (Conchas) & $\begin{array}{l}19^{\circ} 10^{\prime} 57.3^{\prime \prime} \mathrm{N} \\
96^{\circ} 10^{\prime} 51.1^{\prime \prime} \mathrm{W}\end{array}$ & 10 & 0 & 7.47 & 0.250 & $100-250$ & $\begin{array}{l}\text { 75- T. domingensis, U. foliosa, } \\
\text { U. gibba }\end{array}$ \\
\hline
\end{tabular}

$\mathrm{SH}_{1}^{\prime}-\mathrm{SH}_{2}^{\prime}=\sqrt{ }\left(\mathrm{S}_{\mathrm{H}^{\prime} 1}^{2}-\mathrm{S}_{\mathrm{H}^{\prime} 2}^{2}\right)$

La varianza para H' se calculó:

$S_{H}^{2}=\left[\sum \mathrm{f}_{\mathrm{i}} \log ^{2} \mathrm{f}_{\mathrm{i}}-\left(\sum \mathrm{f}_{\mathrm{i}} \log \mathrm{f}_{\mathrm{i}}\right)^{2}(\mathrm{n})^{-1}\right]\left(\mathrm{n}^{2}\right)^{-1}$

Se calculó la diversidad beta como la complementariedad o grado de disimilitud en la composición de especies entre pares, utilizando el índice propuesto por Colwell y Coddington (1995) usando la fórmula $C=(\mathrm{Sj}+\mathrm{Sk}-2 \mathrm{Vjk})$ $(\mathrm{Sj}+\mathrm{Sk}-\mathrm{Vjk})^{-1} * 100$, donde $\mathrm{Sj}$ y $\mathrm{Sk}$ son el número de especies para los sitios $\mathrm{s}$ y j respectivamente, $\mathrm{y}$ Vjk es el número de especies en común entre los sitios.

Análisis de correlación. Se realizó el análisis de correlación no paramétrica de Spearman entre la diversidad (índice de de Shannon) así como entre la riqueza de especies y los parámetros ambientales (salinidad, $\mathrm{pH}$, tamaño, profundidad y número de meses secos). También se realizó una prueba de correlación no paramétrica (Spearman) y un análisis de regresión múltiple (Statistica 6), con los valores del primer eje de la ordenación (PCA) y los valores de cada sitio de los parámetros antes mencionados.

\section{Resultados}

En el Cuadro 1 se describe para cada uno de los lagos las coordenadas, la superficie total en ha, el número de meses que permanecen sin agua, la media anual del $\mathrm{pH}$ y de la salinidad, la profundidad medida en el centro del lago en la época de secas y de lluvias, y el porcentaje de la superficie del espejo de agua cubierta en general por la vegetación (y las especies dominantes). El tamaño es muy variable aunque predominan los cuerpos de agua pequeños, menores a 15 ha. Algunos lagos permanecen inundados todo el tiempo mien- 


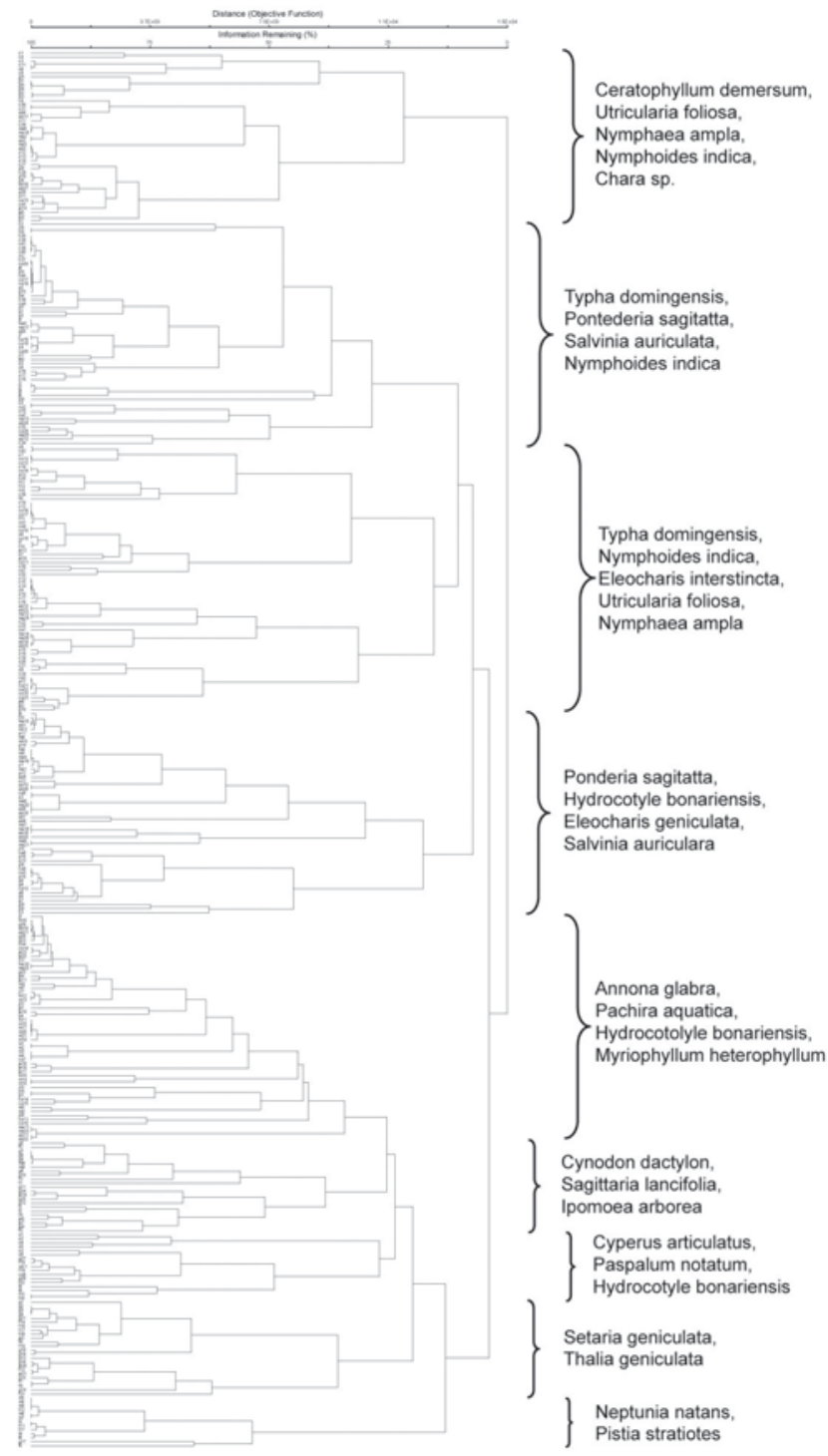

Figura 2. Dendrograma con los 9 grupos florísticos que forman los humedales de los lagos interdunarios..

tras que otros se llegan a secar durante la primavera, como es el caso de Fel, Jag, Tol y Min (las abreviaciones corresponden a los nombres en el Cuadro 1). Cuatro de ellos presentan vegetación en más del $75 \%$ de su superficie, mientras que seis presentan un espejo de agua en más de la mitad de dicha superficie.

Análisis de datos. La clasificación de los lagos con base en todos los cuadros de vegetación muestra nueve grupos (figura 2). El valor de encadenamiento fue de 1.67. El primero de ellos está conformado por la vegetación sumergida (con especies tales como Ceratophyllum demersum, Utricularia foliosa) y la vegetación enraizada de hojas flotantes (con especies tales como Nymphaea ampla y Nymphoides indica).
Se dividieron a un valor menor de similitud, pero se decidió dejarlas juntas. Incluye 43 muestras de 10 lagunas (con mayor número de cuadros en LC, SJ, Man, Abs, Zen y Cat), con 20 especies en total.

El segundo grupo también está formado por dos subgrupos que comparten a la especie Typha domingensis. En un subgrupo Pontederia sagittata es muy frecuente y con alta cobertura, aunque siempre con una dominancia de $T$. domingensis. En el otro subgrupo hay una fuerte presencia de Salvinia auriculata y Nymphoides indica. El grupo está presente en ocho lagunas (LC, SJ, Cat y Zen), conjuntando 56 muestras y 32 especies.

El tercer grupo está formado por dos subgrupos unidos por la presencia de Typha domingensis. En el primero predomina Eleocharis interstincta y Nymphoides indica y en algunos cuadros hay $T$. domingensis. En el segundo subgrupo $T$. domingensis es más abundante y hay una dominancia de Nymphaea ampla y en algunos cuadros Utricularia folio$s a$. Está presente en siete lagunas (principalmente LC, Cat, Col y Zen), con 33 muestras que conjuntan 27 especies.

El cuarto grupo está dominado por Pontederia sagittata e Hydrocotyle bonariensis, aunque en algunos cuadros hay fuerte presencia de Eleocharis geniculata o de Salvinia auriculata. Este grupo está presente en seis lagunas (sobre todo Man, Abs, LP y Tol), e incluyó 51 muestras y 23 especies.

El quinto grupo es sumamente heterogéneo e incluye una gran cantidad de cuadros, algunos de ellos con árboles de selva inundable como Annona glabra y Pachira aquatica. Comparten la presencia con especies herbáceas emergentes, principalmente de Hydrocotyle bonariensis, Cladium jamaisence, Crinum erubescens y Pontederia sagittata, así como en ocasiones la herbácea sumergida Myriophyllum heterophyllum. Incluye muestras de diez lagunas (SJ, LM, Jag, Cat y Man), agrupando 57 muestras y 38 especies. Únicamente se puede determinar la presencia de una selva inundable en LM y restos de esta comunidad en SJ.

El sexto grupo está dominado por Cynodon dactylon, Sagittaria lancifolia, Ipomoea carnea y Setaria geniculata, esta última con baja cobertura en algunos cuadros. La primera especie mencionada y la última han sido introducidas para ganadería en los campos circundantes. Este grupo se presentó en cinco lagunas (DG, Fel y Jag), con 23 muestras y 22 especies. El séptimo grupo está formado por Cyperus articulatus, Paspalum notatum e Hydrocotyle bonariensis, $\mathrm{y}$ algo de Cynodon dactylon en algunos cuadros. Este grupo se registró en seis lagunas (sobre todo en DG, Tol), conjuntando 17 muestras con 25 especies. El penúltimo grupo está dominado de manera importante por Setaria geniculata y como especie acompañante Thalia geniculata. Otras especies que aparecieron en algunos de los cuadros fueron Cyperus articulatus, Cyperus giganteus, y Neptunia natans. Los 24 cuadros pertenecen a tres lagunas (DG, Fel y Jag) y en conjunto presentaron 20 especies. Todos son grupos dominados por especies introducidas para ganadería. 


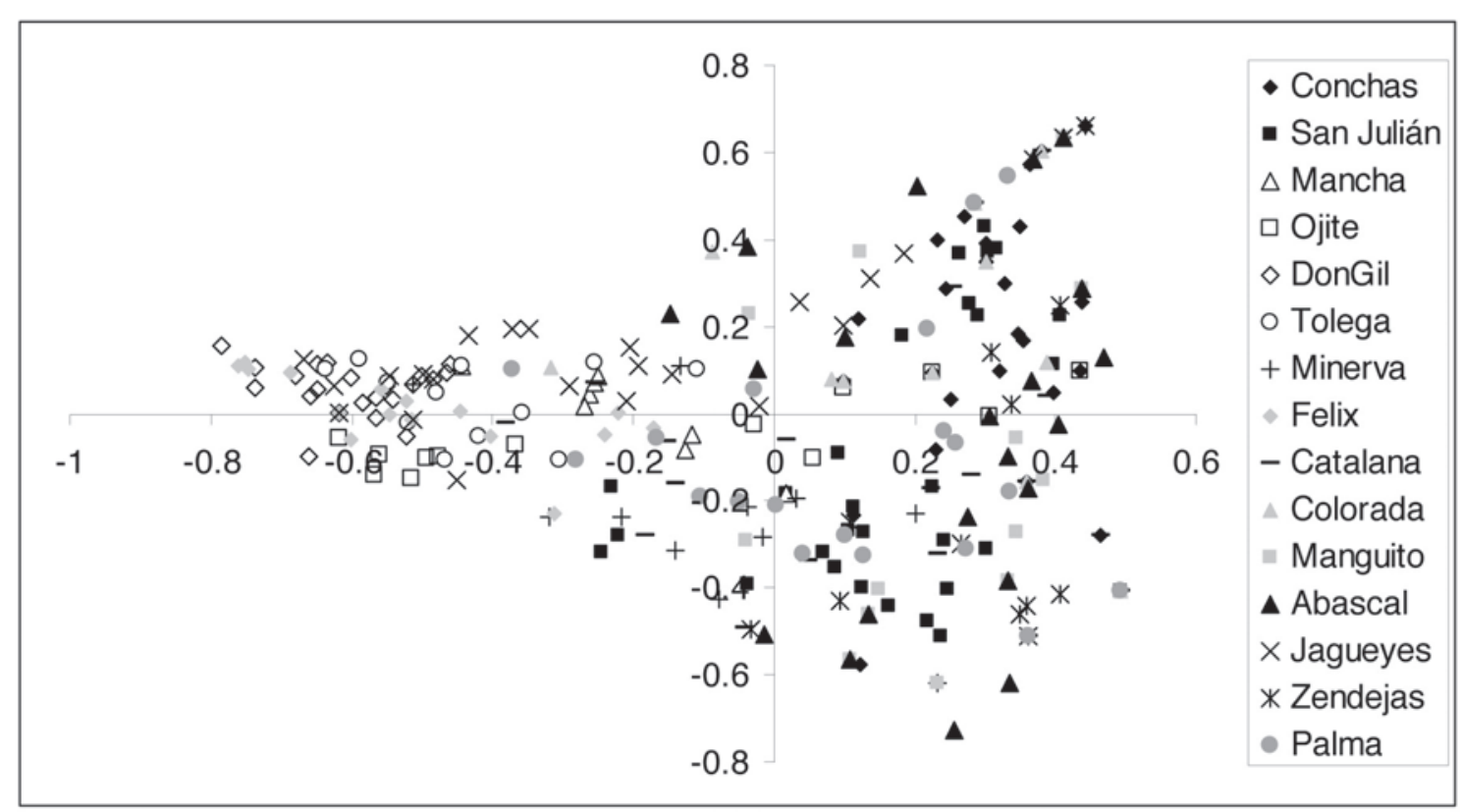

Figura 3. Ordenación por componentes principales con base en todos los cuadros de vegetación, la cual explica entre los dos primeros ejes el 32.2\% de la variación.

El último grupo está formado por las libre flotadoras Pistia stratiotes como dominante y Neptunia natans. Apareció en tres lagunas (sobre todo en LM y Tol donde cubren casi por completo el espejo de agua), con 13 muestras y 7 especies, con muy baja frecuencia y cobertura.

La ordenación de los lagos con base en todos los cuadros de vegetación explica entre los dos primeros ejes $32.2 \%$ de la variación. El eje 1 muestra un gradiente de formas de crecimiento, relacionadas con la degradación del humedal. Las muestras de los lagos de DG, Oj, Tol y Fel se ubican hacia la izquierda del espacio de ordenación (figura 3). Hacia el centro se ubican Jag, Min y LM y hacia la derecha el resto de los lagos, los cuales presentan sobre el eje Y una separación. En la parte superior se encuentran las muestras provenientes de LC y Col y hacia la parte inferior las de Zen, Man y Cat. Mezcladas a todo lo largo de este eje se ubican las muestras de SJ, Abs y LP. Sobre el eje 1, las especies con mayores cargas son Setaria geniculata $(r=-0.3590)$, Cyperus articulatus $(r=-0.2694)$, Neptunia natans ( $r=$ -0.1893), Cynodon dactylon ( $r=-0.2694)$, Pistia stratiotes $(r=-0.1508)$, Sagittaria lancifolia $(r=-0.1477)$, Thalia geniculata $(r=-0.1269)$, Mimosa pigra $(r=-0.1102)$ y con cargas positivas aparecen Typha domingensis $(r=0.5135)$, Utricularia foliosa $(r=0.3120)$, Ceratophyllum demersum $(r=0.2971)$, Nymphaea ampla $(r=0.2108)$, Pontederia sagittata $(r=0.2031)$, Nymphoides indica $(r=0.1680)$ y Salvinia auriculata $(r=0.1216)$. Sobre el segundo eje, las especies con mayores cargas positivas resultaron Utricularia foliosa ( $r=0.4372)$, Ceratophyllum demersum $(r=0.3987)$ y Nymphaea ampla $(r=0.3068)$. Las especies con mayores cargas negativas fueron Pontederia sagittata $(r=-0.5122)$, Typha domingensis ( $r=-0.4281)$, Hydrocotyle bonariensis $(r=-0.1668)$ y Ficus insipida $(r=-0.1118)$.

Los dos primeros ejes de la ordenación de los lagos con base en el valor de importancia de las especies y los datos físicos de los cuerpos de agua, explican el $64.05 \%$ de la variación acumulada (figura 4). Sobre el eje uno, los parámetros ambientales que resultaron más importantes fueron el $\mathrm{pH}$ $(r=-0.754)$ y el número de meses secos (es decir las lagunas que se secan parte del año, $r=0.552$ ). Ello coloca los lagos de Fel, DG, Tol y Jag en el extremo derecho, pues son los que en época de secas pierden el espejo de agua. Las especies con mayores cargas incluyen algunas especies de pastos introducidos tolerantes a la inundación y de especies asociadas con esta actividad: Cynodon dactylon ( $r=0.8971)$, Neptunia natans $(r=0.7453)$, Cyperus articulatus $(r=0.6812)$, Setaria geniculata $(r=0.6742)$, Mimosa pigra $(r=0.6599)$, Asclepias curassavica $(r=0.5894)$, Pennisetum purpureum $(r=0.4980)$, Pistia stratiotes $(r=0.4625)$, Portulaca oleraceae $(\mathrm{r}=0.4507)$, Lippia dulcis $(r=0.4384)$ y Oxalis $\mathrm{sp} .(r=$ 0.4384). En el extremo izquierdo, donde los lagos permanecen inundados todo el año y el $\mathrm{pH}$ es de 6 a 7 , se ubicaron las especies con mayores cargas y fueron Utricularia foliosa $(r=-0.8591)$, Typha domingensis ( $r=-0.6949)$, Ficus insipida $(r=-0.6224)$, Cabomba palaeformis $(r=-0.5547)$, Xanthosoma robustum ( $r=-0.5239)$, Ceratophyllum demersum ( $r=$ -0.5534), Desmodium tortuosum ( $r=-0.4871)$, Chara sp. $(r=$ -0.4837), Melothria pendula ( $r=-0.4837)$, Nymphaea ampla $(r=-0.4689)$, Pachira aquatica $(r=-0.4584)$, Eupatorium odoratum $(r=-0.4237)$ y Paspalum notatum $(r=-0.4126)$. 


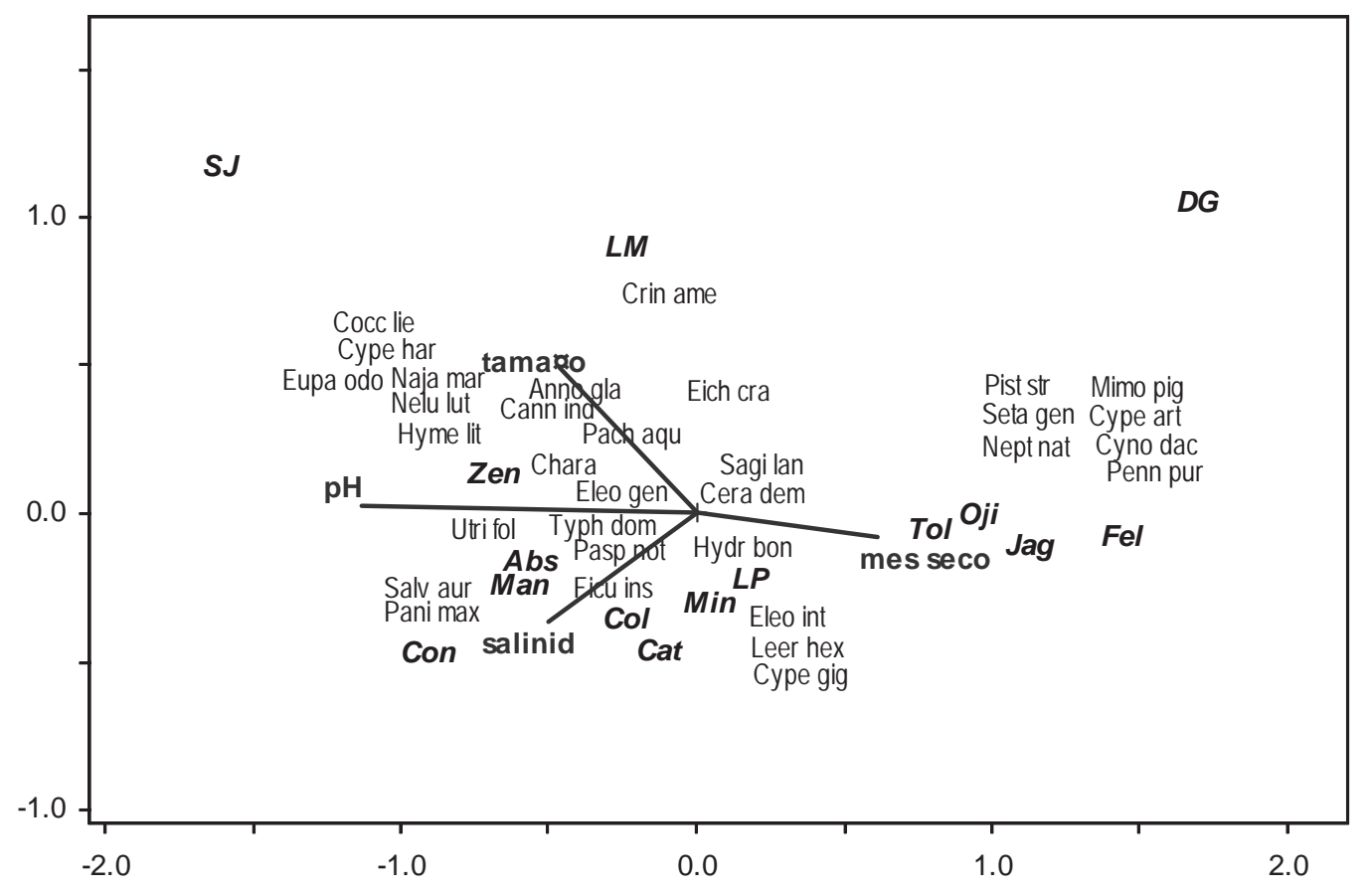

Figura 4. Ordenación por componentes principaleles mostrando la distribución de los lagos con base en los parámetros físicos de los mismos.

En el eje 2, los parámetros que explicaron la mayor variación fueron el tamaño del cuerpo de agua $(r=0.496)$ y la salinidad ( $r=-0.427)$. El lago de mayor tamaño es SJ y los que presentan mayores valores de salinidad son Col, Abs, Man y LP $(0.22,0.25,0.25$ y 0.37 ppm respectivamente). Las especies con mayores cargas positivas fueron Eichhornia crassipes ( $r=0.4793)$, Pachira aquatica $(r=0.4242)$, Ludwigia octovalvis ( $r=0.4115)$ y Diphysa robinioides, Najas marina, Nelumbo lutea, Echinochloa spp., Cyperus hermaphroditus, Hymenocallis littoralis, Ipomoea aquatica, Canna indica, Coccoloba liebmannii y Nectandra salicifolia ( $r=$ $0.2554)$. Las especies con mayores cargas negativas fueron Nymphoides indica ( $r=-0.4964)$, Eleocharis interstincta $(r=$ -0.4937), Leersia hexandra ( $r=-0.3904)$ y Cyperus giganteus $(r=-0.3072)$.

El coeficiente Spearman mostró que el $\mathrm{pH}(\mathrm{R}=-0.67, \mathrm{p}=$ $0.005)$, la profundidad $(\mathrm{R}=-0.803, \mathrm{p}=0.0003)$ y el número de meses secos $(R=0.544, p=0.036)$ están relacionados con los valores del eje 1 de la ordenación. El análisis de regresión no fue significativo para ninguno de los parámetros fisicoquímicos.

Análisis de diversidad. Se registraron 46 familias, 71 géneros y 82 especies, siendo las familias mejor representadas en la zona Cyperaceae (9 especies), Poaceae (7), Fabaceae (6), Convolvulaceae (4), Pontederiaceae (4) y Verbenaceae (3). Del total de familias encontradas, 27 son acuáticas estrictas (Lot et al., 1999), mientras que las demás familias tienen especies de hábitos semiacuáticos o acuáticos. La lista de especies aparece en el apéndice.

La riqueza de especies (S) por lago es variable (Cuadro
2). SJ presentó la mayor riqueza con 33 especies, seguido por Fel con 24 y el que menos especies presentó es LM con solo 5 especies. Los valores más altos del índice de Shannon-Weaver (H') se presentaron en SJ (3.043) seguido por Fel y Oj (2.828 y 2.949). El valor más bajo se dio en LM (1.499); el resto de los lagos presentan valores intermedios. La comparación de los valores de H' de los lagos mostró diferencias significativas (Cuadro 2), mientras que los valores de equitatividad fueron muy homogéneos entre todos los lagos (Cuadro 2), no encontrándose diferencias significativas entre ellos.

El estimador Jacknife detectó que en diez de los lagos los valores estimados de acumulación de especies fueron iguales o muy cercanos a los valores obtenidos en el muestreo. Sin embargo, en las cinco restantes (Fel, LP, Zen, Man y Con) no se obtuvo la asíntota, ni en lo muestreado ni con los estimadores (Cuadro 2). La complementariedad entre pares de lagos (diversidad beta) tuvo valores desde 0.33 hasta 1.0 (Cuadro 3). El lago LM tuvo valores mayores de 0.90 por lo que se diferenció de la mayoría de los otros lagos. Los lagos Fel, DG, Jag, Tol, Oj, Min, Cat, Abs, Man y Con tuvieron valores que fluctuaron entre 0.70 y 0.89 , compartiendo una tercera parte de sus especies. Man, Abs y Zen compartieron el $50 \%$ con valores entre 0.50 y 0.69 . Cat y Col compartieron aproximadamente el $75 \%$.

Correlación. La correlación que se realizó entre las variables ambientales versus la diversidad (índice de Shannon) y la riqueza de especies es no significativa. Se considera que este resultado obedece a que las distribuciones encontradas en la ordenación son multifactoriales. 
Cuadro 2. Valores de riqueza, equitatividad y diversidad para cada lago estudiado. Las letras a la derecha del índice de Shannon indican diferencias significativas (prueba de t Hutchenson).

\begin{tabular}{|c|c|c|c|c|}
\hline Clave & Riqueza de especies & Equitatividad & Índice de Shannon $\left(\mathrm{H}^{\prime}\right)$ & Jacknife de primer orden \\
\hline LM & 5 & 0.96 & $1.49 \mathrm{a}$ & 5 \\
\hline$\overline{D G}$ & 20 & 0.94 & $2.57 \mathrm{dc}$ & 14.9 \\
\hline Fel & 24 & 0.94 & $2.82 \mathrm{e}$ & 19.42 \\
\hline Jag & 15 & 0.97 & $2.53 \mathrm{dc}$ & 14 \\
\hline Tol & 10 & 0.98 & $1.97 \mathrm{~b}$ & 8.9 \\
\hline Oj & 23 & 0.95 & $2.94 \mathrm{e}$ & 21.92 \\
\hline Cat & 15 & 0.96 & $2.39 \mathrm{dc}$ & 19.6 \\
\hline Col & 10 & 0.98 & $2.04 \mathrm{~b}$ & 11.8 \\
\hline SJ & 33 & 0.94 & $3.04 \mathrm{e}$ & 45.68 \\
\hline LP & 13 & 0.97 & $2.35 \mathrm{c}$ & 19.7 \\
\hline Min & 15 & 0.94 & $2.37 \mathrm{C}$ & 16.6 \\
\hline Zen & 15 & 0.95 & $2.33 \mathrm{C}$ & 20.6 \\
\hline Abs & 13 & 0.95 & $2.32 \mathrm{c}$ & 17.97 \\
\hline Man & 13 & 0.94 & $2.21 \mathrm{bc}$ & 15.75 \\
\hline Con & 21 & 0.93 & $2.47 \mathrm{dc}$ & 29.76 \\
\hline
\end{tabular}

\section{Discusión}

Los resultados obtenidos confirman que los lagos interdunarios son cuerpos de agua someros, temporales o permanentes, de agua dulce y tamaño variable, en los cuales se establecen distintos tipos de humedales, desde los dominados por especies herbáceas emergentes (popales y tifales) hasta selvas inundables.

La composición florística de los humedales se puede agrupar en nueve grandes grupos, con características de humedales poco alterados (los primeros cinco grupos) hasta los que están siendo fuertemente impactados (los cuatro restantes). Los grupos seis, siete y ocho (figura 2) tienen una fuerte presencia de especies de pastos introducidos para el forrajeo de ganado vacuno (Cynodon dactylon, Setaria geniculata, Paspalum notatum, Pennisetum purpureum), actividad dominante en las dunas aledañas a algunos de los lagos (Obs. Pers.). También aparecen algunas especies consideradas como secundarias y características de sitios perturbados (Sida rhombifolia, Crotalaria incana) y aparecen en combinación con especies características de humedales, aunque estas últimas en proporciones bajas (Sagittaria lancifolia, Thalia geniculata, Hydrocotyle bonariensis). Este arreglo de las especies muestra que los lagos de DG, Oji, Tol, Fel y Jag presentan numerosas especies de pastos mezcladas con la vegetación hidrófita, producto tanto de la ganadería extensiva que se da a su alrededor como de la desecación de los mismos lagos debido probablemente a la erosión producida por el ganado y el crecimiento de la frontera agropecuaria. También cabe decir que estos lagos se encuentran muy cercanos entre si y probablemente pertenecen a un sistema hidrológico regional, donde no se acumula tanta agua por escurrimiento subsuperficial como en los otros casos (García-Calderón y de la Lanza-Espino, 2002; Arriaga et al., 2000).
Cuatro especies de hidrófitas son consideradas como malezas (Eichhornia crassipes, Pistia stratiotes, Salvinia minima y $S$. auriculata) y provienen de Centro y Sudamérica (Wetzel, 1981; Cronk y Fennessy, 2001; Dodds, 2002). De manera general su presencia no implica necesariamente una eutrofización de los lagos dado que la cobertura e importancia dentro de cada lago fue baja, pero su dominancia puede estar evidenciando una alteración del ambiente. Diferentes autores (Gersberga et al., 1986; Seoánez-Calvo, 1999; EPA, 2000, Helgen y Gernes 2001; Wissinger et al., 2001; Scheffer, 2001; Cronk y Fenessy 2001) las consideran junto con Typha, como filtros biológicos que regulan las concentraciones de nutrientes y sólidos suspendidos totales en la columna de agua. LM es un lago con fuerte dominancia de Pistia stratiotes. Durante muchos años anidaron en los bordes de selva inundable de Annona glabra y Pachira aquatica que lo rodean, gran cantidad de individuos de garzas de varias especies (observación personal). Así mismo recibe el escurrimiento subsuperficial de las aguas de los terrenos de dunas y sembradíos de caña de azúcar sobre dunas antiguas de los alrededores (Yetter, 2004). Ello incrementó de manera importante la cantidad de nitratos $\left(0.873 \mathrm{mgL}^{-1}\right)$ en el agua de este lago (datos no publicados).

La riqueza de especies es muy heterogénea en los lagos interdunarios. Lot (1991) reporta para los principales ambientes acuáticos del estado de Veracruz un total de 67 familias y 166 especies y de éstas, solo 32 familias y 33 especies se encuentran en los lagos interdunarios. Considerando solo las familias y especies que son estrictamente acuáticas, Lot y Ramírez-García (1998) reportan para el estado de Veracruz 51 especies de las cuales el $45 \%$ se encuentran en estos sistemas y el $20 \%$ de las especies reportadas a nivel nacional (Lot et al. 1999). Ello significa que a pesar de que la mayoría de estos sistemas son de dimensiones pequeñas (1-15 ha), conjuntan un alto núme- 
Cuadro 3. Valores de complementariedad o grado de disimilitud en la composición de especies obtenidos entre los 15 lagos, expresados en porcentajes. Se obtuvieron mediante el índice de Colwell y Coddington (1955).

\begin{tabular}{|c|c|c|c|c|c|c|c|c|c|c|c|c|c|c|c|}
\hline LM & LM & & & & & & & & & & & & & & \\
\hline DG & 96 & DG & & & & & & & & & & & & & \\
\hline Fel & 100 & 74 & Fel & & & & & & & & & & & & \\
\hline Jag & 100 & 79 & 66 & Jag & & & & & & & & & & & \\
\hline Tol & 93 & 80 & 78 & 81 & Tol & & & & & & & & & & \\
\hline $\mathrm{Oj}$ & 96 & 73 & 72 & 73 & 73 & Oj & & & & & & & & & \\
\hline Cat & 100 & 97 & 81 & 85 & 75 & 64 & Cat & & & & & & & & \\
\hline $\mathrm{Col}$ & 100 & 96 & 90 & 86 & 75 & 73 & 33 & Col & & & & & & & \\
\hline SJ & 94 & 91 & 90 & 93 & 95 & 85 & 80 & 83 & SJ & & & & & & \\
\hline LP & 100 & 93 & 80 & 78 & 79 & 71 & 66 & 72 & 85 & LP & & & & & \\
\hline Min & 100 & 87 & 74 & 85 & 75 & 77 & 69 & 80 & 85 & 72 & Min & & & & \\
\hline Zen & 100 & 97 & 92 & 85 & 96 & 84 & 69 & 61 & 76 & 72 & 88 & Zen & & & \\
\hline Abs & 100 & 93 & 88 & 92 & 95 & 80 & 72 & 78 & 79 & 76 & 78 & 66 & Abs & & \\
\hline Man & 100 & 100 & 91 & 92 & 90 & 83 & 72 & 78 & 82 & 70 & 78 & 66 & 55 & Man & \\
\hline Con & 100 & 97 & 93 & 87 & 88 & 90 & 75 & 69 & 79 & 82 & 83 & 87 & 77 & 86 & Cor \\
\hline
\end{tabular}

ro de familias y especies. Los valores de diversidad alfa más altos se presentan en 1) los lagos de mayor superficie y que no se secan y que por tanto mantienen humedales más diversos y conservados (SJ y Con), y con un nivel de perturbación bajo $(<20 \%)$ en sus alrededores por las actividades agropecuarias y 2 ) en aquellos en que se han combinado especies de hidrófitas y especies consideradas como secundarias propias de los campos con actividades agropecuarias (DG, Jag, Fel y Oj) (figura 4 y cuadro 3). LM es el lago con menor valor de diversidad, posiblemente por la dominancia de Pistia stratiotes que no permite el crecimiento de ninguna otra especie en el cuerpo de agua y por estar rodeado en tres lados de selva inundable que produce sombra e inhibe el crecimiento de especies que requieren luz solar directa.

La hipótesis de picos o valores mayores de diversidad en estados intermedios de perturbación parece presentarse en el caso de los humedales de los lagos interdunarios rodeados de actividades agropecuarias. Esta hipótesis plantea que cuando los disturbios se presentan, la competencia entre especies es alta solo en raras ocasiones y por tanto se gana diversidad (Rosenzweig, 1995; Petraitis et al., 1989). Se ha reportado una mayor riqueza de especies en situaciones intermedias de disturbio (Connell, 1978; Guo, 1996; Pollock et al., 1998; Weiher, 2003). Se ha encontrado tanto para humedales, como para pastizales y otros ecosistemas, que los valores más altos de riqueza, se obtienen en situaciones intermedias respecto a la biomasa (Tilman, 1982; Rodríguez et al., 1987; Huston, 1994; Weiher, 2003).

Boorman et al. (1997) y Leentvaar (1997), mencionan un total de 60 especies que corresponden a 27 familias en los lagos interdunarios del noreste de Europa. En nuestro caso los sistemas son más diversos ya que se encontraron un total de 80 especies en 44 familias. Wilcox (1995) menciona solo 20 especies para los sistemas interdunarios de los Grandes Lagos de Estados Unidos, lo que indica que en estos ambientes el estado de Veracruz es más biodiverso que los sitios mencionados por los autores antes mencionados.

La complementaridad de especies entre los humedales de estos lagos (cuadro 3) muestra que son bastante diferentes entre sí. Entre todos ellos conjuntan una gran cantidad de especies aunque hay diferencias en composición y estructura entre ellos. Constituyen cuerpos de agua aislados por condiciones muy distintas, ya que las dunas de arena son ambientes con muy baja capacidad de retención de agua, pobres en nutrientes y en algunos de ellos con arena móvil (Moreno-Casasola, 1982). Ello hace que los lagos permanezcan aislados y que la colonización dependa de aves, otro tipo de fauna visitante o del ser humano.

El tipo de comunidades de humedales que se establece en los lagos interdunarios no son privativos de estas zonas. Constituyen humedales herbáceos y arbóreos que encuentran su hábitat en una estructura geomorfológica muy particular, las depresiones en los sistemas de dunas costeras alimentados por el manto freático. Se deben desarrollar planes de manejo junto con los propietarios que permitan el uso de estos lagos pero que al mismo tiempo garanticen la permanencia de la composición y la estructura de las hidrófitas características de estos lagos someros.

\section{Agradecimientos}

A CONACYT por la beca No.164477, al Instituto Tecnológico de Veracruz por la beca-comisión otorgada al primer autor, SEMARNAT (2002-C-01-0190), Canadian International Development Agency (S-061870), Instituto de Ecología A.C. (902-17). A A. Lot Helgueras, M. Olvera, G. Castillo, T. Mejía Saulés, M. Palacios Ríos, E. Cejudo Espinosa, D. Infante Mata y C. Durán. Agradecemos especialmente los comentarios hechos al manuscrito por A.C. Travieso Bello y H. López Rosas y el apoyo de Graciela Sánchez y Rosario Landgrave. 


\section{Literatura citada}

Arriaga C.L., Aguilar S.V. y Alcocer D.J. 2000. Aguas continentales y diversidad biológica de México. CONABIO. México.

Boorman L.A., Londo G. y van der Maarel E. 1997. Communities of dune slacks and wet plains. En: van der Maarel E. Ed. Dry coastal ecosystems: general aspects (Ecosistems of the world 2C), pp.275-296. Elsevier. Amsterdam.

Colwell R.K. 2005. EstimateS v7.5.: statistical estimation of species richness and shared species from samples. <viceroy.eeb. uconn.edu/EstimateS>

Colwell R.K. y Coddington J.A. 1995. Estimating terrestrial biodiversity through extrapolation. En: Hawksworth D.L. Ed. Biodiversity. Measurement and estimation, pp101-118. Chapman \& Hall-The Royal Society. London.

Connell J.H. 1978. Diversity in tropical rain forest and coral reefs. Science 199:1302-1310.

Cronk J.K.y Fennessy M.S. 2001. Wetland plants. Biology and ecology. Lewis Publishers. New York.

de Bruyn G.J. 1997. Animal communities in dutch dune area. En: van der Maarel E. Ed. Dry coastal ecosystems: general aspects (Ecosistems of the world 2C), pp.361-386. Elsevier. Amsterdam.

Dodds W.K. 2002. Freshwater ecology: Concepts and environmental applications. Academic Press. San Diego, California.

Domínguez J.M.L. y Bittencourt A.C.S.P 1994. Utilização de padrões de sedimentação costeira como indicadores paleoclimáticos naturais (proxies). Revista Brasileira de Geociencias 24:312.

EPA [United States Environmental Protection Agency]. 2000. Manual: Constructed Wetlands Treatment of Municipal Wastewaters. EPA. Cinacinnati, Ohio. Disponible en linea: <www. epa.gov/nrmrl/pubs/625r99010/625r99010.pdf> (consultado: 8 octubre 2009)

García E. 1981. Modificaciones al sistema de clasificación climática de Köppen. Instituto de Geografía, Universidad Nacional Autónoma de México, México D. F.

García-Calderón J.L. y de la Lanza-Espino G. 2002. Las aguas epicontinentales de México. En: de la Lanza-Espino.G. y GarcíaCalderón J.L. Eds. Lagos y presas de México, pp.5-34. AGT Editor, México.

Gersberg R.M., Elkins B.V., Lyon S.R. y Goldman C.R. 1986. Role of aquatic plants in wastewater treatment by artificial wetlands. Water Research 20:363-368.

Grootjans A.P., Adema E.B., Bekker R.M. y Lammerts E.J. 2004. Why coastal dune slacks sustain a high biodiversity. En: Martínez M.L. y Psuty N.P. Eds. Coastal dunes: Ecology and conservation (Ecological Studies vol. 171), pp. 85-101. SpringerVerlag. Berlin.

Guo Q. 1996. Effects of bannertail kangaroo rat mounds on smallscale plant community structure. Oecologia 106:247-256.

Helgen J.C. y Gernes M.C. 2001. Monitoring the condition of wetlands: Index of biological integrity using invertebrates and vegetation. En: Rade R.B., Batzer D.P. y Wissinger S.A. Eds. Bioassessment and management of North American freshwater wetlands, pp.167-185. John Wiley \& Sons. New York.

Huston M.A. 1994. Biological diversity: the coexistence of species on changing landscape. Cambridge, University Press. Cambridge.

Lammerts E.J. y Grootjans A.P. 1998. Key environmental variables determining the occurrence and life span of basiphilous dune slack vegetation. Acta Botanica Neerlandica 47:369-392.

Leentvaar P. 1997. Communities of dune lakes. En: van der Maarel, Ed. Dry coastal ecosystems: general aspects (Ecosistems of the world 2C), pp.297-322. Elsevier. Amsterdam.

Lot A. 1991. Vegetación y flora vascular acuática del estado de Veracruz. Tesis de doctorado. Facultad de Ciencias, Universidad Nacional Autónoma de México, México, D.F. 226 pp.

Lot A., Novelo A. y Ramírez-García P. 1993. Diversity of mexican aquatic vascular plant flora. En: Ramamoorthy T.P., Bye R., Lot A. y Fa J. Eds. Biological diversity of Mexico: Origins and distribution, pp.577-591. Oxford University Press. Nueva York.

Lot A., Novelo A., Olvera M. y Ramírez-García P. 1999. Catálogo de angiospermas acuáticas de México. Cuadernos del Instituto de Biología vol. 33, Instituto de Biología. Universidad Nacional Autónoma de México.México, D.F.

Magurran A.E. 2004. Measuring biological diversity. Blackwell Publishing. Oxford, UK.

Martínez M.L., Moreno-Casasola P. y Vázquez G. 1997. Effects of disturbance by sand movement and inundation by water on tropical dune vegetation dynamics. Canadiam Journal of Botany 75:2005-2014.

McCune B. y Grace J.B. y Urban D.L. 2002. Analysis of ecological communities. MjM Softweare Desing. Gleneden Beach. Oregon.

Moreno-Casasola P. 1982. Ecología de la vegetación de dunas costeras: factores físicos. Biotica 7:577-602.

Moreno-Casasola P. y Espejel I. 1986. Classification and ordination of coastal sand dune vegetation along the Gulf and Caribbean Sea of Mexico. Vegetatio 66:147-182.

Moreno-Casasola P. y Vázquez G. 1999. The relationship between vegetation dynamics and water table in tropical dune slacks. Journal of Vegetable Science 10:515-524.

Moreno-Casasola P. 2004. A case study of conservation and management of a tropical sand dune system: La Mancha-El Llano. En: Martínez M.L. y Psuty N.P. Eds. Coastal dunes: Ecology and conservation (Ecological Studies vol. 171), pp. 319-334. Springer-Verlag. Berlin.

Peet R.K. 1975. Relative diversity indices. Ecology 56:496-498.

Peralta-Pelaez L.A., Deloya C. y Moreno-Casasola P. 2007. Insectos acuáticos asociados a las lagunas interdunarias de la región central del estado de Veracruz, México. Neotropical Entomology 36:342-355.

Petraitis P.S., Latham R.E. y Niesenbaum R.A. 1989. The maintenance of species diversity by disturbance. The Quartely Review of Biology 64:393-418.

Pollock M.M., Naiman R.J. y Hanley T.A. 1998. Plant species richness in riparian wetlands a test of biodiversity theory. Ecology 79:94-105.

Queiroz-Matias L. y Paula-Nunes E. 2001. Levantamento floristicos da área de proteção ambiental de Jericoacoara, Ceará. Acta Botanica Brasilica 15:35-43.

Rodríguez M.A., Álvarez J., Pascual M.R. y Gómez-Sal A. 1987. Variaciones en la estructura aérea y subaérea de pastos de montaña según el grado de aprovechamiento. Pastos 17:348-362.

Ranwell D.S. 1972. Ecology of salt marshes and sand dunes. Chapman and Hall, Londres.

Rosenzweig M.L. 1995. Species diversity in space and time. Cambridge University Press. Cambridge.

Sarabia, B.C. 2004. Sistema lagunar de la ciudad de Veracruz: propuesta de manejo bajo la visión de proyectación y gestión 
ambiental. Tesis Doctoral. Colegio de Posgraduados, Campus Veracruz. Universidad de Chapingo. México. 325pp.

Scheffer M. 2001. Ecology of shallow lakes. Kluwer Academic Publisher. Netherlands.

Seoánez-Calvo M. 1999. Aguas residuales: tratamiento por humedales artificiales: Fundamentos científicos, Tecnología, Diseño. Mundi-Prensa. Madrid.

Siemens A.H., Moreno-Casasola P. y Sarabia-Bueno C. 2006. The metabolization of dunes and wetlands by the city of Veracruz, Mexico. Journal of Latin American Geography 5:7-29.

Sival F.P. y Lammerts E.J. 1997. Acid neutralization in dune slack soils: influence on longevity of basiphilous communities. En: Sival F.P. Dune soil acidification threatening rare plants species, pp.9-25. Tesis Doctoral, Universidad de Groningen, Groningen, Holanda.

Sival F.P. y Strijkstra-Kalk M. 1997. Atmospheric deposition of acidifying and eutrophicating substances and its effects on dune slack vegetation En: Sival F.P. Dune soil acidification threatening rare plants species, pp.27-40. Tesis Doctoral, Universidad de Groningen, Groningen, Holanda.

Tilman D. 1982. Rosource competition and community structure. Princeton University Press. Princeton. New Jersey.

Tiner R.W. 2003. Geographically isolated wetlands in the United States. Wetlands 23:494-516.

van der Meulen F. y Naber F.R.M. 1997. Dune water catchment in the Netherlands. En: van der Maarel Ed. 1997. Dry coastal ecosystems: general aspects (Ecosistems of the world 2C), pp.533556. Elsevier. Amsterdam.

Vázquez G. 2004. The role of algal mats on community succession in dunes and dune slacks. En: Martínez M.L. y Psuty N.P. Eds. Coastal dunes: Ecology and conservation (Ecological Studies vol. 171), pp.189-204. Springer-Verlag. Berlin.

Weiher E. 2003. Species richness along multiple gradient: testing a general multivariate model in oak savannas. Oikos 101:311-316.

Westhoff V. y van der Maarel E. 1978. The Braun-Blanquet approach. En: Whittaker R.H. Ed. Classification of plant communities, pp.287-397. Dr W. Junk b.v. Publishers-The Hague. Netherland.

Wetzel R.G.1981. Limnología. Omega. Barcelona.

Wilcox D.A. 1995. Wetland and aquatic macrophytes as indicator of anthropogenic hydrologic disturbance. Natural Areas Journal 15:240-248.

Wissinger S.A., Ingmire S.G. y Bogo J.L. 2001. Plant and invertebrate communities as indicators of success for wetlands restored for wildlife. En: Rade R.B., Batzer D.P. y Wissinger S.A. Eds. Bioassessment and management of North American freshwater wetlands, pp. 207-236. John wiley \& Sons. Nueva York.

Yetter J.Ch. 2004. Hydrology and geochemistry of freshwater wetlands on the gulf coast of Veracruz, México. Thesis Master of Science. University of Waterloo, Ontario, Canada. 168pp.

Zar J.H. 1999. Bioestatistical analysis. Prentice Hall. New Jersey.

Recibido: 11 de mayo del 2009

Aceptado: 14 de octubre del 2009 
Apéndice 1. Lista de familias y especies registradas en los humedales de los lagos interdunarios de la zona rural del centro de Veracruz.

\section{CHAROPHYTA}

Characeae

Chara sp.

\section{MAGNOLIOPHYTA}

\section{Alismataceae}

Echinodorus andrieuxii (Hook. \& Arn.) Small.

Sagittaria lancifolia L.

Sagittaria latifolia Willd.

\section{Amaryllidaceae}

Crinum americanum Hort.Prag. ex Steud.

Hymenocallis littoralis Salisb.

\section{Annonaceae}

Annona glabra L.

\section{Apiaceae}

Hydrocotyle bonariensis Lam.

\section{Araceae}

Pistia stratiotes L.

Xanthosoma robustum Schott.

\section{Asclepiadaceae}

Asclepias curassavica L.

\section{Asteraceae}

Eupatorium odoratum L.

Melampodium divaricatum DC.

\section{Bombacaceae}

Pachira aquatica Aubl

Cabombaceae

Cabomba palaeformis Fassett.

\section{Cannaceae}

Canna indica L.

Ceratophyllaceae

Ceratophyllum demersum L.

\section{Convolvulaceae}

\section{Aniseia martinicensis Coisy}

Ipomoea alba L.

Ipomoea aquatica Forssk.

Ipomoea carnea Jacq.

Melothria pendula L.

\section{Cyperaceae}

Carex sp.

Cladium jamaicense Crantz,

Cyperus articulatus L.

Cyperus giganteus Vahl

Cyperus hermaphroditus Standl.

Eleocharis geniculata (L.) Roem. \& Schult.

Eleocharis interstincta (Vahl) Roem. \& Schult.

Eleocharis parvula (Roem. \& Schul) Palla

Fuirena camptotricha Wright in Sauvalle.

Rhynchospora colorata (Hitchcock.) H. Pfeiff.

\section{Euphorbiaceae}

Croton sp.

\section{Fabaceae}

Crotalaria incana L.

Desmodium tortuosum (Sw.) DC.

Diphysa robinioides Benth \& Oerst.

Mimosa pigra L.

Neptunia natans Druce.

Phaseolus sp.

\section{Haloragaceae}

Myriophyllum heterophyllum Minchx.

\section{Lauraceae}

Nectandra salicifolia Nees.

\section{Lemnaceae}

Lemna sp.

\section{Lentibulariaceae}

Utricularia foliosa L.

Utricularia gibba L.

Limnocharitaceae

Limnocharis flava Buchenau.

Malvaceae

Sida rhombifolia L.

Marantaceae

Thalia geniculata L.

Menyanthaceae

Nymphoides indica (L.) Kuntze.

\section{Moraceae}

Brosimum alicastrum Sw.

Ficus insipida subsp. scabra C.C.Berg

\section{Najadaceae}

Najas marina L.

Nelumbonaceae

Nelumbo lutea Pers.

Nymphaeaceae

Nymphaea ampla DC.

Onagraceae

Ludwigia octovalvis (Jacq.) P.H.Raven.

Orchidaceae

Bletia purpurea (Lam.) DC.

Oxalidaceae

Oxalis sp.

\section{Poaceae}

Cynodon dactylon (L.) Pers.

Echinochloa pyramidalis (Lam.) Hitch.\& Chase.

Echinochloa sp.

Leersia hexandra Sw.

Panicum maximum Jacq.

Paspalum notatum Flüggé.

Pennisetum purpureum Schum.

Setaria geniculata P.Beauv. 
Apéndice 1. Continuación

\section{Polygonaceae}

Coccoloba liebmannii Lindau.

\section{Pontederaceae}

Heteranthera limosa Willd.

Eichhornia crassipes Sloms

Pontederia sagittata C.Presl.

Portulacaceae

Portulaca oleracea L.

Rubiaceae

Crusea sp.

Sapindaceae

Serjania sp.

Solanaceae

Solanum diphyllum L.

Sterculaceae

Helicteres sp.

\section{Typhaceae}

Typha domingensis Pers.

\section{Verbenaceae}

Citharexylum berlandieri S.Watson

Lippia alba N.E.Br. ex Britton \& P.Wilson

Lippia dulcis Trevir.

\section{PTERIDOPHYTA}

Pteridaceae

Acrostichum aureum L.

\section{Salviniaceae}

Salvinia auriculata Aubl.

Salvinia minima Baker.

Thelypteridaceae

Thelypteris interrupta (Willd.) K. Iwats 